\title{
Canceladores de Eco
}

\author{
Edson Benedicto R. Féris
}

Neste trabalho analisa-se o funcionamento de canceladores de eco adaptativos. Estuda-se a otimização do controle de adaptação do dispositivo ao meio de transmissão. Discutem-se, também, os diagramas específicos de alguns fabricantes desse dispositivo.

\section{Introdução}

A transformação de um sistema de dois para quatro fios é necessária para a transmissão de sinais telefônicos pelos métodos de multiplexagem analógica ou digital. Esta trans. formação se faz utilizando-se dispositivos denominados junções híbridas. Entretanto. a utilização destes dispositivos acarreta o aparecimento de retorno do sinal, ou seja, eco, devido ao desequilíbrio dos mesmos. Este desequilíbrio acontece em razão da impossibilidade de se manter as impedâncias de todas as linhas tronco e de assinantes com um valor igual e imutável para todas elas.

Este problema se tornou mais efetivo na era das comunicações via satélite, pois o intervalo entre o sinal incidente e o refletido fica maior que meio segundo. Isto faz com que a comunicação telefônica se torne altamente desagradável.

Para resolver o problema do eco tentou-se, inicialmente, a interrupção do caminho de retorno durante a conversa do assinante $A$, conforme esquematizado na Fig. 1 , Quando

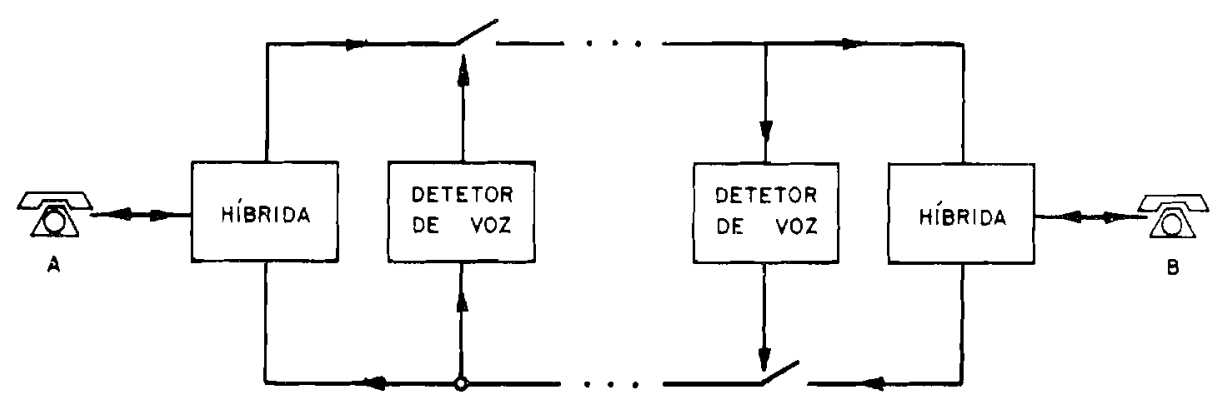

Figura 1. Diagrama básico do supressor de eco.

autor é Pesquisador do CPqD.TELEBRÁS, Caixa Postal 1579, 13.100, Campinas, SP.

evista da Sociødade Brasileira de Telecomunicações

olume 1, No 1, novembro 1986 
o assinante A falava, o circuito de retorno era desligado. A decisão era feita por um detetor de nível de sinal no circuito de ida. Este sistema recebeu o nome de supressor de eco. Entretanto, seu funcionamento sempre foi passível de críticas. Para grandes desequilíbrios da junção híbrida, confundiam-se os sinais de A e B. Isto causava distúrbios inaceitáveis à comunicação. Além disto, mesmo na situação de equilíbrio normal, tinha-se eco na primeira sílaba da conversa do assinante $A$, pois sua energia era utilizada para a decisão do chaveamento do circuito de retorno.

Somente com o desenvolvimento dos canceladores de eco [1] $-[5]$ é que se pode considerar resolvido satisfatoriamente o problema em questão. Além do emprego de canceladores de eco para a finalidade descrita, existe uma tendência atual de utilização do seu princípio de funcionamento, nos circuitos de digitalização de linhas de assinantes.

\section{Princípio de Funcionamento}

Nesta seção, descreve-se o princípio de funcionamento dos canceladores de eco. Fundamentalmente, parte-se do sinal incidente e procura-se sintetizar um sinal idêntico ao eco. Este sinal, que chamaremos de eco artificial, é subtraído do eco verdadeiro, resultando, teoricamente, um eco nulo.

O arranjo básico está esquematizado na Fig.2. Dado um assinante distante A, o circuito que cancelaria seu eco fica junto à híbrida do lado do assinante B. Este será denominado de assinante próximo. Para cancelar o eco do assinante $B$ deve ser instalado um outro circuito próximo à híbrida do lado do assinante $A$.

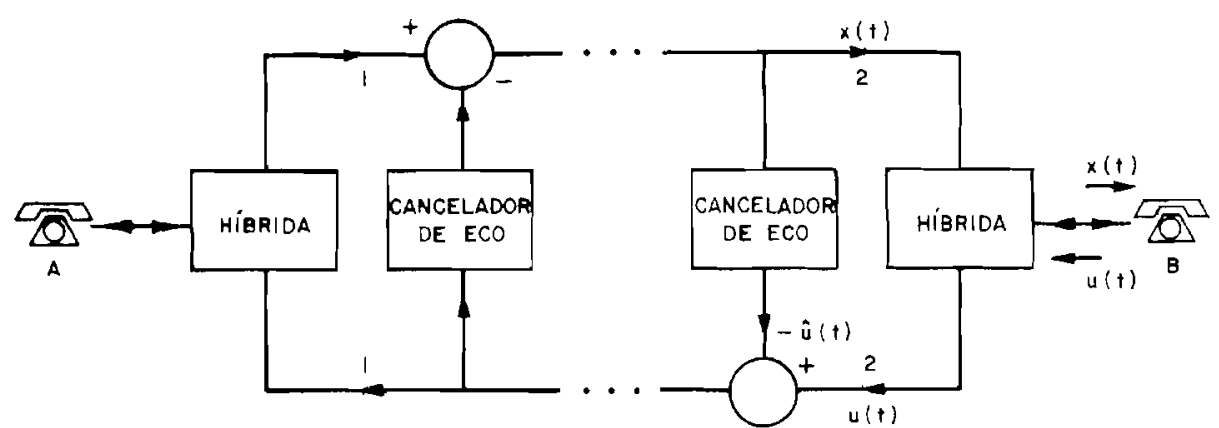

Figura 2.Diagrama básico do canceladior de eco.

Este arranjo minimiza o tempo de atraso entre o sinal incidente $x(t)$ e seu eco $u(t)$. Desta maneira, em uma ligação via satélite não entraria, neste atraso, aquele corres. pondente às distâncias até o satélite, pois estas distâncias estariam compreendidas entre 1 e 2 na Fig. 2 . 
Normalmente se coloca o cancelador de eco junto à híbrida da central de trânsito de maior hierarquia. Com isto minimiza-se a quantidade de canceladores de eco nos sistemas telefônicos. Entretanto isto acarreta, muitas vezes, a presença de várias centrais de comutação entre esta híbrida e o assinante próximo B. Isto significa que nem sempre ele está tão próximo. Um cancelador de eco típico prevê um atraso de retorno de até $25 \mathrm{~ms}_{\text {s }}$ Isto corresponde a distâncias de até $2.500 \mathrm{~km}$. Algumas vezes existe possibilidade para suportar um atraso até quatro vezes maior.

A Fig. 3 mostra o eco produzido por uma excitação impulsiva. $O$ tempo de atraso $T_{0}$ depende da instalação. Entretanto, a duração $T_{d}$ da dispersão é sempre da ordem de $2 \mathrm{~ms}$ a $3 \mathrm{~ms}$.

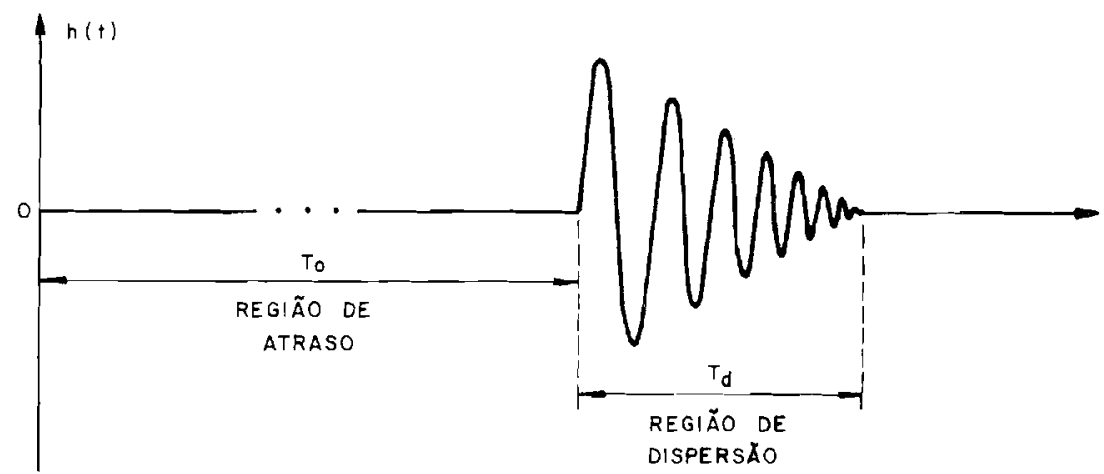

Figura 3. Eco de uma excitação impulsiva.

\section{Teoria de Funcionamento}

Considera-se agora, através dos diagramas apresentados nas figuras 4 e 5, a teoria de funcionamento de um cancelador de eco.

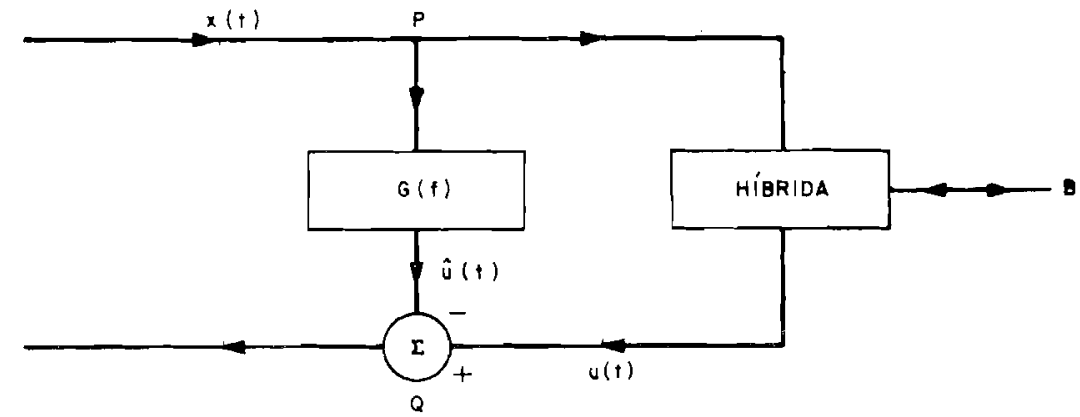

Figura 4. Diagrama analítico.

levista da Sociedade Brasileira de Telecomunicações 


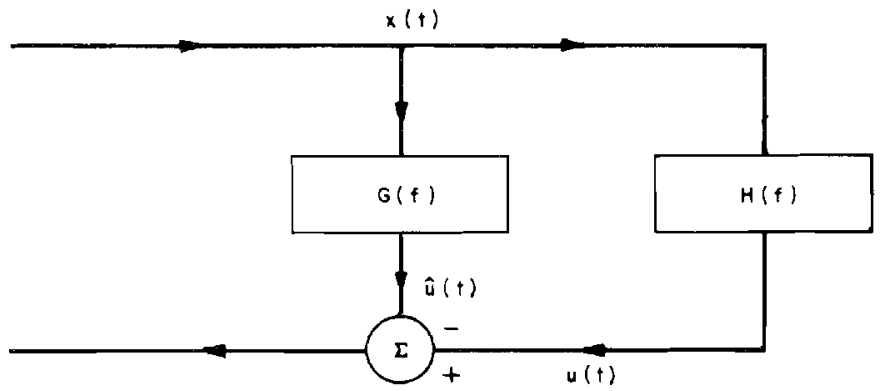

Figura 5. Diagrama equivalente.

Com referência ao diagrama da Fig. 4, vamos supor que se conhece a resposta impulsiva do caminho PBO. Neste caso tem-se, para qualquer excitação $x(t)$,

$$
u(t)=h * x(t)
$$

onde $\mathrm{h}(\mathrm{t})$ é a resposta impulsiva do caminho $\mathrm{PBO}$ e onde o símbolo * representa a operação de convolução.

Trabalhando com as transformadas de Fourier destas funções do tempo, resulta

$$
U(f)=H(f) \cdot X(f)
$$

onde $U(f), H(f)$ e $X(f)$ são as transformadas de Fourier do sinal de retorno, da resposta impulsiva do percurso PBO e do sinal de excitação, respectivamente. Desta maneira,

$$
u(t)=F^{-1}[H(f) . X(f)]
$$

onde $\mathrm{F}^{-1}$ representa a transformada de Fourier inversa.

A partir destas considerações chega-se à sugestão primária para cancelamento de eco. Determina-se a resposta ao impulso do circuito ida-retorno. Sintetiza-se um filtro cuja resposta ao impulso $g(t)$ seja igual a $h(t)$. Na saída deste filtro tem-se um sinal $\hat{u}(t)$ que, teoricamente, seria igual a $u(t)$. Faz-se a subtração entre $u(t)$ e $\hat{u}(t)$ e se tem o cancelamento de eco.

\section{Implementação}

A maneira mais conveniente de se sintetizar um filtro, a partir de sua resposta ao impulso, é pela técnica de filtros transversais. Nestes filtros, sintetiza-se o sinal $\hat{u}(t)$ através de 
$\hat{u}(t)=g_{1} \times(t)+g_{2} \times\left(t-T_{s}\right)+g_{3} \times\left(t-2 T_{s}\right)+\ldots+g_{n} \times\left[t-(n-1) T_{s}\right]$

onde $x\left(t-K T_{s}\right)$ representa o sinal $x$ atrasado de $K$ períodos de amostragem $T_{s}$. Os parâmetros $g_{j}$ são coeficientes e tanto o período $T_{s}$ como a quantidade $n$ de parcelas são determinados em função da duração e do espectro de freqüência da resposta impulsiva a ser sintetizada. Os coeficientes são calculados a partir da sistemática de projetos de filtros transversais.

É possível reescrever (1) simplificadamente, como

$$
\hat{u}(t)=g_{1} x_{1}+g_{2} x_{2}+\ldots g_{n} x_{n}
$$

onde $x_{i}$ corresponde ao sinal $x$ atrasado de $(i-1)$ períodos $T_{S^{*}}$ Pode-se escrever (2) na forma matricial

$$
\tilde{u}(t)=G^{\top} \mathbf{X}
$$

onde $\mathrm{G}$ e Xsão matrizes $\mathrm{n} \times 1$ dadas por

$$
G=\left[\begin{array}{c}
g_{1} \\
g_{2} \\
\cdot \\
\cdot \\
\cdot \\
g_{n}
\end{array}\right] \quad \text { e } \quad x=\left[\begin{array}{c}
x_{1} \\
x_{2} \\
\cdot \\
\cdot \\
x_{n}
\end{array}\right]
$$

A Fig. 6 mostra o diagrama de implementação do filtro em questão.

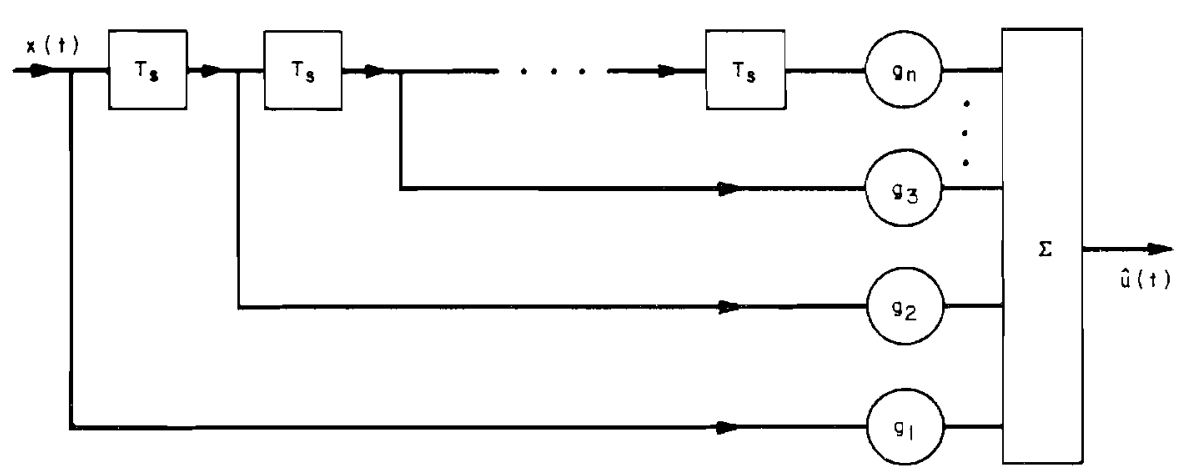

Figura 6. Diagrama do filtro transversal.

Revista da Sociedade Brasileira de Telecomunicações

Volume 1, NO 1, novembro 1986. 
Embora este dispositivo possa ser construído a partir de atrasadores, multiplicadores e somador analógicos, a tecnologia atual o implementa na forma digital. Trabalha-se com amostras de $x(t)$ quantizadas digitalmente. $O$ tempo $T_{s}$ é o periodo de relógio. Os atrasadores são registradores de deslocamento. As multiplicações dos coeficientes e a soma final são realizadas pela técnica de aritmética lógica.

O sistema aqui proposto apresenta algumas dificuldades práticas. $O$ verdadeiro caminho de ida e volta que produz o eco não é perfeitamente constante. Além das mudanças óbvias ocasionadas por mudanças de ligações, ou por conexões e desconexões de extensões durante a conversação, ou transferência de chamadas via "key-system", existem ainda variações lentas de ganho e outras flutuações da função de transferência do caminho de eco. Podemos, então, concluir que o cancelamento do eco só seria efetivo se houvesse um meio de se adaptar constantemente o filtro transversal à resposta real do eco.

Em meados da década de 60, J.L. Kelly Jre, dos Laboratórios da Bell, propôs um sistema adaptativo que foi mais tarde modificado por Sondhi [1] e mais recentemente por Duttweiler [3].

\section{Teoria de Funcionamento do Cancelador Adaptativo de Kelly e Sondhi}

Supondo que $u(t)$ fosse o sinal real de eco e que se conhecesse a verdadeira matriz de coeficientes de um filtro que reproduzisse $u(t)$, teriamos

$$
u(t)=H^{\top} \cdot x
$$

onde

$$
H=\left[\begin{array}{c}
h_{1} \\
h_{2} \\
\vdots \\
h_{n}
\end{array}\right]
$$

seria a matriz de coeficientes exata do caminho de eco.

Devemos supor que para todo $i h_{i}$ é quase estacionário, isto é, sua variação é extremamente lenta com relação à duração da resposta impulsiva. Neste caso

$$
\frac{d H}{2} \cong 0
$$


Após a subtração entre os sinais do eco verdadeiro e o artificial restaria o sinal de erro $\mathrm{e}(\mathrm{t})$ dado por

$$
e(t)=u(t)-\hat{u}(t)=H^{\top} \cdot X-G^{\top} \cdot X=(H-G)^{\top} \cdot X=R^{\top} \cdot X
$$

onde

$$
\mathbf{R}=\mathbf{H}-\mathbf{G}
$$

Vamos fechar o elo de controle utilizando como sinal de correção uma determinada função do erro

$$
F(e)=F\left(R^{\top} \cdot \mathbf{X}\right)
$$

Para que haja convergência, isto é, para que o erro se anule, é suficiente que $F(e)$ seja ímpar e monótona não decrescente, conforme está demonstrado a seguir. Algumas funções de erro satisfazendo estas condições são apresentadas na Fig. 7.

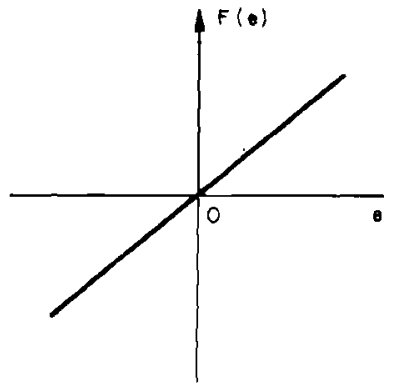

(a)

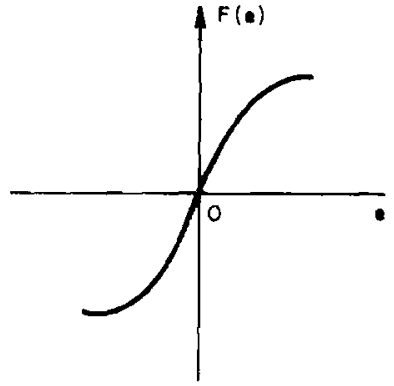

(b)

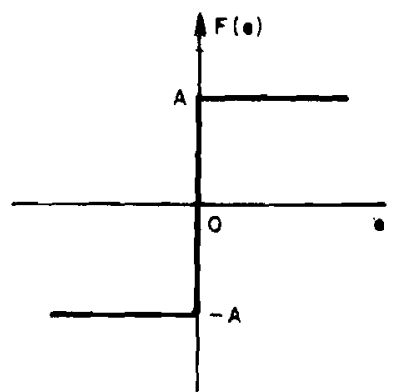

(6)

Figura 7. Alguns exemplos de $F(e)$ que provocam convergência do controle.

Vamos determinar a matriz de coeficientes $\mathbf{G}$, de tal maneira que satisfaça a equação diferencial

$$
\frac{d G}{d t}=K \cdot F(e) \cdot x
$$

ou

$$
\frac{d G}{d t}=K \cdot F\left(R^{\top} \cdot X\right) \cdot X
$$

onde $K$ é um ganho escolhido.

De (5) tem-se $\mathbf{G}=\mathbf{H}-\mathbf{R}$ e portanto 


$$
\frac{d G}{d t}=\frac{d H}{d t}-\frac{d R}{d t} \cong-\frac{d R}{d t}
$$

Multiplicando-se os dois membros de (6) por $-2 \mathbf{R}^{\top}$ tem-se

$$
-2 R^{\top} \cdot \frac{d G}{d t}=-2 R^{\top} \cdot K F\left(R^{\top} \cdot X\right) X
$$

Substituindo (7) em (8) resulta

$$
2 \mathbf{R}^{\top} \cdot \frac{\mathrm{dR}}{\mathrm{dt}}=-2 \mathbf{R}^{\top} \cdot \mathrm{KF}\left(\mathbf{R}^{\top}, \mathbf{X}\right) \mathbf{X}=-2 \mathrm{~K} \mathbf{R}^{\top}, \mathbf{X} F\left(\mathbf{R}^{\top}, \mathbf{X}\right)
$$

Mas

$$
2 \mathbf{R}^{\top} \cdot \frac{d \mathbf{R}}{d t}=\frac{d\left(\mathbf{R}^{\top} \cdot \mathbf{R}\right)}{d t}
$$

e portanto

$$
\frac{d\left(R^{\top} \cdot R\right)}{d t}=-2 K R^{\top} \cdot X F\left(R^{\top} \cdot X\right)=-2 K e F(e)
$$

Para $F(e)$ impar e monótona não decrescente sabemos que e.F(e) será sempre positiva ou nula. Portanto o segundo membro de (9) será sempre negativo lou zero quando $\mathrm{e}=\mathbf{0 )}$. Portanto $\left[\mathrm{d}\left(\mathbf{R}^{\top} \cdot \mathbf{R}\right) / \mathrm{dt}\right]<0$ para $\mathrm{e} \neq 0 \in\left[\mathrm{d}\left(\mathbf{R}^{\top} \mathbf{R}\right) / \mathrm{dt}\right]=0$ para $\mathrm{e}=0$.

Como a derivada de $\mathbf{R}^{\top}$. $\mathbf{R}$ é negativa ou nula conclui-se que $\mathbf{R}^{\top} . \mathbf{R}$ é decrescente quando e $\neq 0$ e tem inclinação nula quando $e=0$. Note que $\mathbf{R}^{\top}$. $\mathbf{R}$ corresponde ao quadrado do comprimento do vetor $\mathbf{R}=\mathbf{H}-\mathbf{G e}$, portanto, $\left[\mathrm{d}\left(\mathbf{R}^{\top} . \mathbf{R}\right) / \mathrm{dt}\right]<0$ significa que o comprimento $\mathbf{H}-\mathbf{G}$ é decrescente. Agora, prova-se que o valor de $R^{\top} . \mathbf{R}$ para o caso e $=0$, também é nulo. Com efeito, $\operatorname{como} \mathbf{R}=\mathbf{H}-\mathbf{G}$, se o erro se anula é porque todos os coeficientes $h_{i}$ são iguais aos $g_{i}$ correspondentes, anulando a matriz $R$ e sua transposta.

Conclui-se assim que a presença de um sinal de erro e(t) diferente de zero faz a expressão $\mathbf{R}^{\top} \cdot \mathbf{R}$ decrescer até zero. Neste estado final tem-se $e(t)=0$.

\section{Implementação do Cancelador Adaptativo}

Do que foi exposto, concluímos que o cancelador adaptativo a ser implementado deve satisfazer (6), ou seja

$$
\frac{d G}{d t}=K F\left(R^{\top} \cdot X\right) X=K F((H-G) T \cdot X) X=K F(u(t)-\hat{u}(t)) X
$$


A implementação do cancelador adaptativo correspondente é apresentada na Fig. 8.

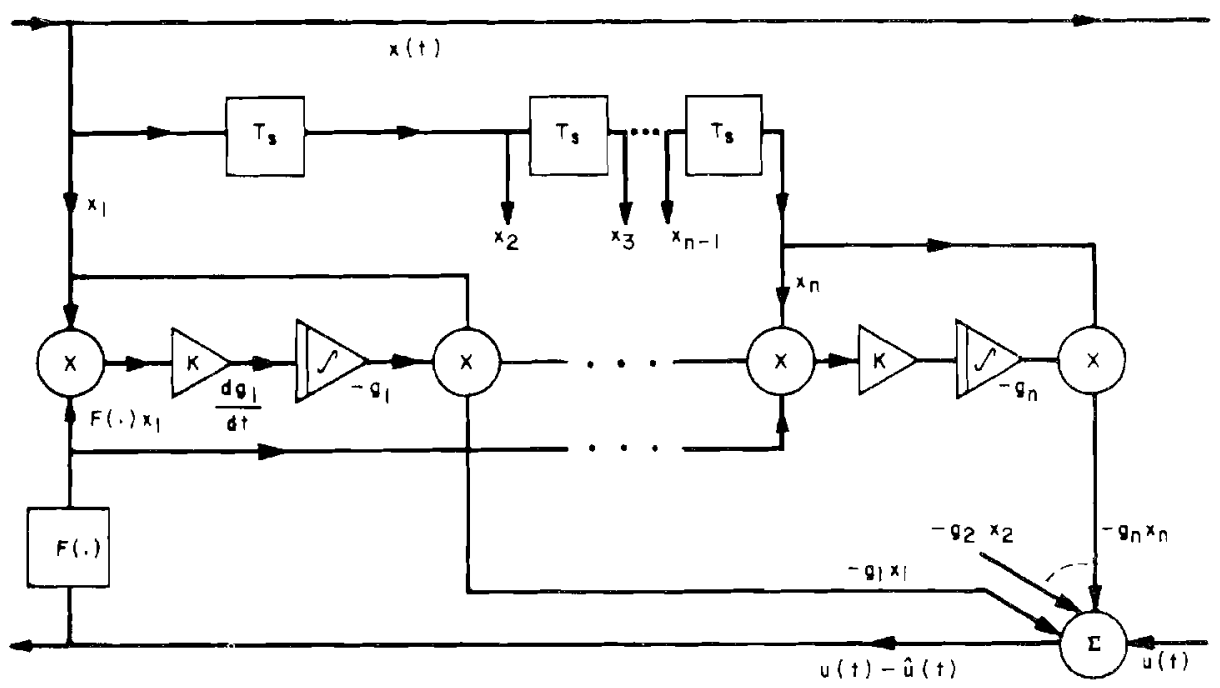

Figura 8. Cancelador adaptativo de eco.

Observe-se de (6) que a velocidade de convergência depende diretamente do ganho $K$. Ainda a partir de (6), vemos que a velocidade de convergência cresce também com a amplitude do sinal $x$ e com $F(e)$. O ideal é que a velocidade fosse constante, acar. retando um tempo de correção da ordem de $0,5 \mathrm{~s}$.

No que se refere à escolha da função $F$, vimos que ela deve ser ímpar. A proposição original de Kelly seria a reta $F(e)=$ e ou $F\left(\mathbf{R}^{\top}, \mathbf{X}\right)=\mathbf{R}^{\top} . \mathbf{X}$, conforme mostrado na $\mathbf{F i g}$. $7(a)$. Neste caso tem-se de $(6)$

$$
\frac{d G}{d t}=K\left(R^{\top} \cdot \mathbf{X}\right) \mathbf{X}
$$

o que indica que a velocidade de correção é proporcional ao quadrado da amplitude do sinal incidente, ou seja, proporcional à potência média $P_{m}$ deste sinal. Tem-se então

$$
\frac{d \mathbf{G}}{d t}=K \text { a } P_{m}
$$

snde a é um vetor de coeficientes de proporcionalidade. Isto faria com que uma và. iação por um fator 10 na amplitude do sinal provocasse uma variação por um fator 100 na velocidade de convergência dos coeficientes $g_{i}$.

evista da Sociedade Brasileira de Telecomunicações 
Sondhi [1] propôs a utilização de um limitador ideal para o qual $F(e)=A s g n(e)$, onde $\operatorname{sgn}(e)=1$ se $e \geqslant 0$ e $\operatorname{sgn}(e)=-1$ se $e<0$, conforme mostrado na Fig. $7(\mathbf{c})$. Tem-se então

$$
\frac{d G}{d t}=\operatorname{KAsgn}\left(R^{\top} . X\right) X
$$

Como $\operatorname{sgn}\left(\mathbf{R}^{\top}, \mathbf{X}\right)$ depende apenas do sinal algébrico de $\mathbf{R}^{\top} . \mathbf{X}$, conclui-se que a velo. cidade de convergência dependerá linearmente da amplitude do sinal. Desta forma, uma variação na amplitude do sinal por um fator 10 acarreta uma variação na velocidade de convergência também por um fator 10 .

Finalmente, Duttweiler [3] propôs em 1978 uma espécie de CAG (controle automático de ganho) no amplificador de ganho $K$, que compensaria a variação de amplitude do sinal. Neste caso pode-se adotar, por simplicidade, $F(e)=$ e. $O$ dispositivo calcula a média dos quadrados dos sinais $x_{i}$. Desta forma, tem-se uma grandeza proporcional à potência média do sinal $x$. A seguir, a amplitude de saída do amplificador de ganho $K$ é dividida pelo valor daquela grandeza. Resulta portanto

$$
\frac{d G}{d t}=K \cdot \frac{1}{P_{m}} \cdot a \cdot P_{m}=K a
$$

Atualmente, praticamente todos os canceladores de eco funcionam por este método de controle da velocidade de convergência.

\section{Influência de Ruído e Interferências}

Vamos supor que, junto com o sinal $u(t)$ de eco, tem-se também um ruido $n(t)$. Neste caso, chamaremos $V(t)$ a composição dos dois sinais, ou seja

$$
y(t)=u(t)+n(t)
$$

A diferença entre este sinal e o eco artificial será então

$$
e(t)=y(t)-\hat{u}(t)=u(t)+n(t)-\hat{u}(t)=R^{\top} \cdot x+n(t)
$$

Repetindo-se o mesmo tratamento matemático anterior teríamos

$$
\frac{d\left(R^{T} ; B\right)}{d t}=K R^{T}, X F\left(R^{T} \cdot X+n(t)\right)
$$

Neste caso, se $F$ é ainda a função sgn, conclui-se que o lado direito só será negativo caso $R^{\top}$. $X+n(t)$ tenha o mesmo sinal algébrico que $R T$ ? $X \dot{E}$ interessante notar que $n(t)$ pode representar, além do ruído presente no circuito, o sinal transmitido por $B$ isola- 
damente ou durante uma conversação dupla. Durante esta última situação, se supusermos que $\mathrm{n}(\mathrm{t})$ tem média zero, então concluiremos que o sinal algébrico da média de $R^{T} . X+n(t)$ é igual àquele da média de $R^{T} . X$. Portanto, desde que o tempo de convergência seja suficientemente grande ( $\mathrm{da}$ ordem de $0,5 \mathrm{~s}$ ), o controle ainda converge. Entretanto, durante a presença única da conversa de $\mathrm{B}$, o controle poderia provocar distúrbios no funcionamento do sistema devido à utilização de falsa referência.

Para contornar esta dificuldade adotou-se o congelamento dos coeficientes durante a presença do sinal $B$. Como seu sinal é mais forte que o eco (especialmente devido à ação do cancelador), é feita a deteção de sua presença. Então interrompe-se o controle, mantendo-se os valores dos $\mathrm{g}_{j}$ estacionários até o momento em que $A$ volta a falar sem a presença da conversa de $B$.

Para esta deteção usa-se o algoritmo de Geigel, referenciado por Duttweiler [3]

$$
y_{i}>\alpha \cdot \max \left[x_{i-1}, x_{i-2}, \ldots, x_{i-n}\right]
$$

O fator $\alpha$ é utilizado para compensar as atenuações no caminho do eco. A comparação de niveis deve ser feita com as amostras precedentes para levar em consideração o tempo de atraso da informação.

\section{Desempenho do cancelador de eco}

O sistema descrito tem a possibilidade de reduzir o sinal do eco tipicamente para $20 \mathrm{~dB}$ [1] abaixo do nivel original. Particularmente, quando o filtro é implementado utilizando amostras de sinal quantizadas digitalmente, o ruído de quantização pode piorar o cancelamento de 1 a $2 \mathrm{~dB}[3]$. Para muitas situações isto é considerado insu. ficiente. Por isto usa-se o dispositivo "center-clipping" (limitador central). Ele atua na situação em que apenas A está falando e, portanto, o cancelador está ativo, fazendo com que o sinal de eco esteja reduzido ao pequeno nível mencionado. O limitador central produz a rejeição deste pequeno sinal. Desta forma o assinante $A$ não conseguirá ouvir seu eco, uma vez que sua eliminação atinge $60 \mathrm{~dB}[3]$, [5] com a presença deste novo dispositivo, para o qual o relacionamento entre entrada e saída é apresentado na Fig. $\mathbf{9}$.

Como não é de se esperar que durante a presença da voz de B ocorram grandes alterações na resposta do circuito de ida e retorno, concluimos que os coeficientes $g_{i}$, mesmo congelados, permanecem com valores aceitáveis para o cancelamento do eco.

Em resumo, durante a conversação dupla a redução de eco será tipicamente de $20 \mathrm{~dB}$, passando a $60 \mathrm{~dB}$ quando $A$ fala sozinho, uma vez que, neste caso, atua também o limitador central.

Revista da Sociedade Brasileira de Telecomunicaçōes 


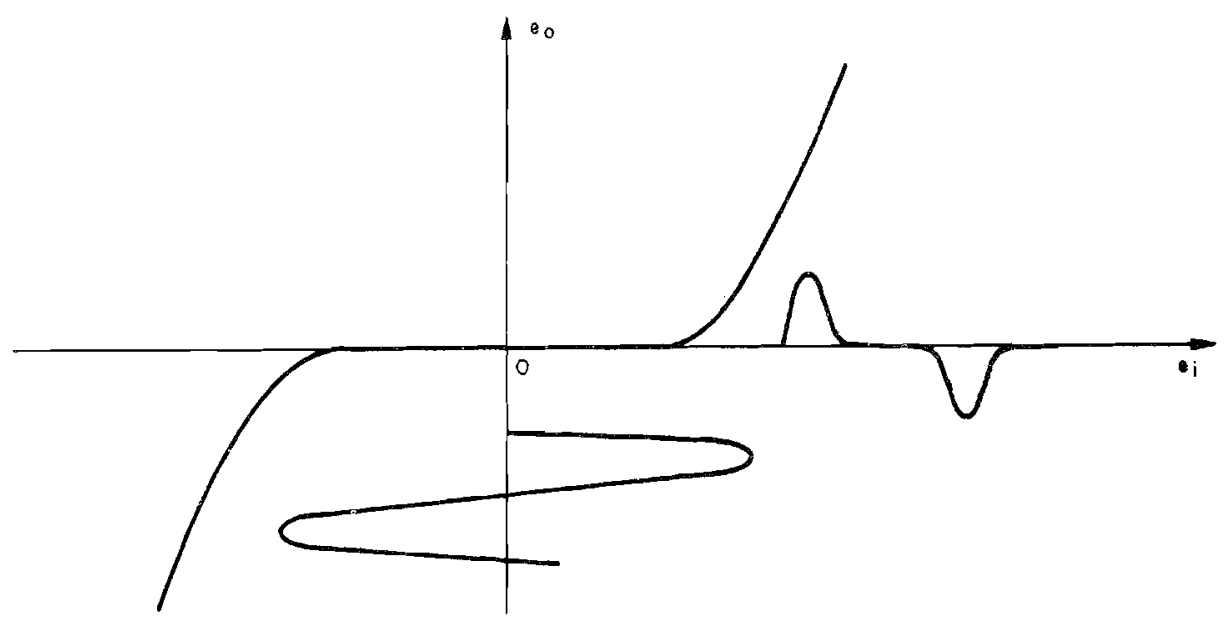

Figura 9. Limitador central.

\section{Considerações sobre a Implementação do Cancelador de Eco na Forma Digital}

A implementação do cancelador de eco na forma digital é esquematizada na Fig. 10. Quando se parte de um sinal PCM ("Pulse Code Modulation") monocanal comprimido (compressão no processo de compansão), a primeira operação é retirar as amostras quantizadas $x_{i}$. Isto se faz por meio de um conversor série-paralelo e pela expansão de niveis (linearização). Estas amostras lineares são entregues ao filtro digital adaptativo. Em sua saída temos amostras do sinal artificial de eco ûi com sinal algébrico trocado.

Em outro ramo do circuito, o eco verdadeiro, na forma PCM comprimido, também é convertido para paralelo e linearizado, Deste modo, tem-se as amostras reais de eco $u_{i}$.

A seguir é realizada a soma algébrica destas amostras com as amostras $-\hat{U}_{i}$, resultando amostras de erro $e_{j}$. Estas amostras são introduzidas no sistema de controle adaptativo dos coeficientes do filtro digital.

Finalmente, as amostras de saída do somador são comprimidas e convertidas para o formato série, ou seja, colocadas na forma PCM monocanal.

Devemos, ainda, mencionar a presença dos dispositivos de interrupção do controle adaptativo (congelamento) e de acionamento do "center-clipping". Ambos os dispositivos são realizados pela técnica de lógica digital.

Os Laboratórios da Bell produzem um circuito integrado VLSI ("very large scale integration") que contém as funções descritas a menos do conversor série-paralelo. Ele 


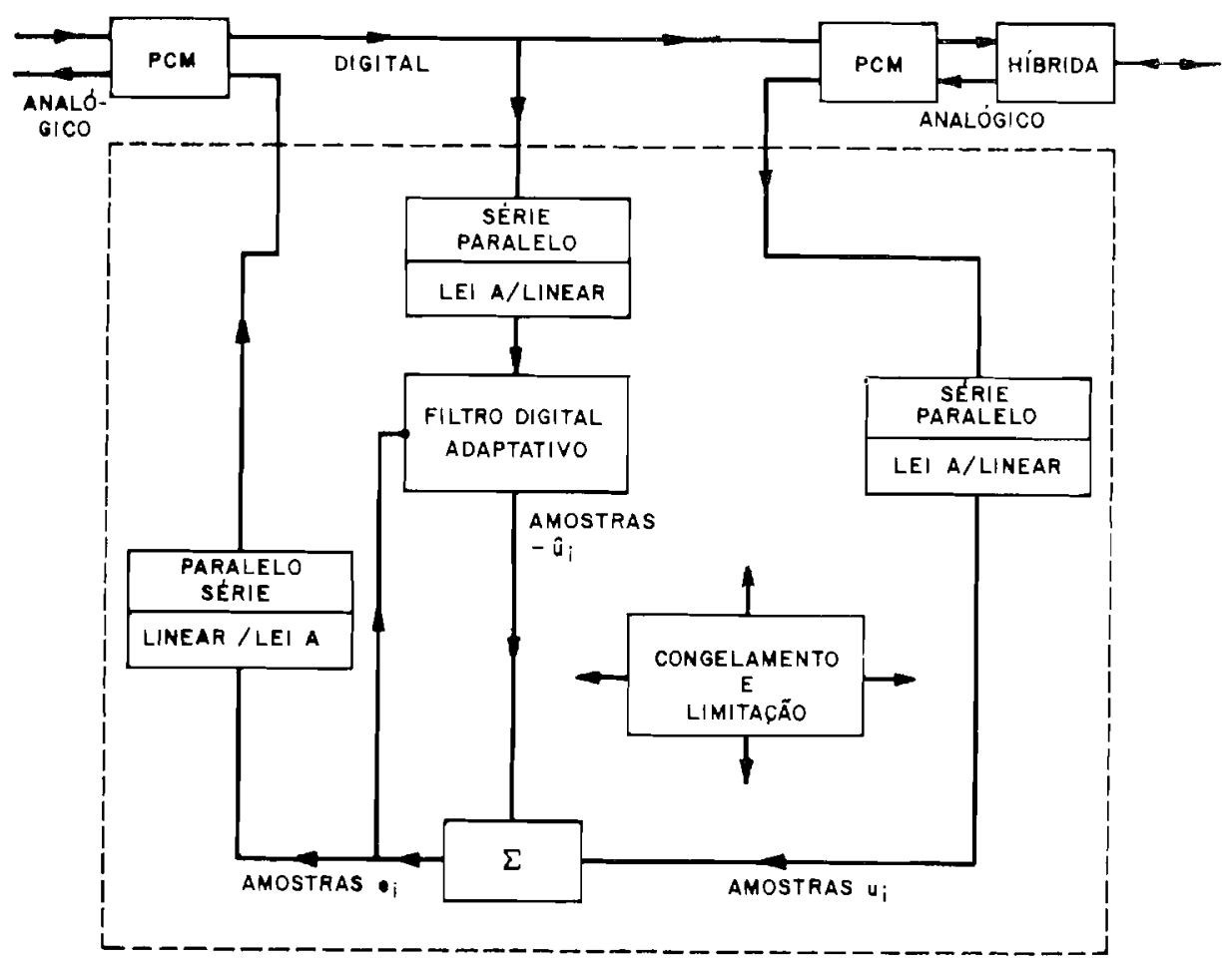

Figura 10. Implementação digital do cancelador de eco.

recebe as amostras $x_{i}$ na forma comprimida pela lei $\mu-255$ e fornece as amostras de saída na mesma forma. Caso a linha seja analógica deve-se acrescentar codecs (codificador-decodificador) incluindo compansão pela lei $\mu-255$,conforme indicado na Fig. 10. Este circuito integrado, entretanto, não é fornecido para outros fabricantes de equipamentos.

A empresa AMI (American Microsystems, Inc.) comercializa o circuito integrado S2816, que é um cancelador de eco adaptativo para um canal PCM, lei $\mu-255$ [5].

Para a construção de um cancelador de eco completo é necessária a utilização de, pelo menos, mais quatro circuitos integrados especiais fornecidos pela própria AMI, a saber: S68A52 - conversor série-paralelo;

S6810 - memória RAM ("random access memory") que armazena as amostras por um período equivalente ao atraso do eco;

$\mathrm{S} 6802$ - microprocessador que monitora e controla todas as operações;

S6846 - memoria ROM ("read only memory"), controlador de entrada e saída, gerador de fases de relógio e dispositivo que armazena o programa do mi. croprocessador.

Revista da Sociedade Brasileira de Telecomunicaçð̃es

Volume 1, N! 1, novembro 1986. 
Pode ainda ser fornecido pela AMI o circuito integrado S3507, que é um codec PCM com lei de compansão $\mu-255$.

Do exposto concluímos que, para o nosso caso, os circuitos da AMI ou da Bell não podem ser usados em linha P.CM, uma vez que no Brasil adotou-se a lei $A$ de com. pansão. Entretanto, estes sistemas são transparentes para linhas analógicas. Se quisermos utilizá-los para linhas PCM é necessário adicionar-se memórias ROM ou EPROM ("erasable programmed read only memory") de conversão de lei $\mu-255$ para lei $A e$ vice-versa.

\section{Apêndice A}

\section{Determinação do Número de Atrasadores do Filtro Digital}

A partir do teorema de Nyquist e pelos padrões telefônicos usuais, decidiu-se utilizar, para o canal de áudio de $3,4 \mathrm{kHz}$, a freqüência de amostragem de $8 \mathrm{kHz}$. Isto corresponde a um período $T_{\mathrm{s}}$ de amostragem de $125 \mu \mathrm{s}$.

Na técnica de filtros transversais é necessário utilizar uma quantidade de atrasadores tal que o atraso total tenha duração igual ou maior que a resposta impulsiva que se quer sintetizar.

De acordo com os comentários feitos em conexão com a Fig. 3, para uma distância de até $2.500 \mathrm{~km}$, a duração do sinal de eco inclui um atraso $T_{0}$ máximo de $25 \mathrm{~ms}$ e uma dispersão $T_{d}$ da ordem de $2 \mathrm{~ms}$ a $3 \mathrm{~ms}$, perfazendo uma duração máxima total de $28 \mathrm{~ms}$. Portanto, o número de atrasadores será

$$
n=\frac{28}{0,125} \cong 224
$$

O circuito integrado da. Bell utiliza 127 atrasadores e igual número de circuitos de controle adaptativo de coeficientes $g_{i}$ que compreendem multiplicadores e integradores, conforme o diagrama da Fig. 8. Isto corresponde a uma distância máxima de funcionamento da ordem de $1.500 \mathrm{~km}$.

Entretanto, devido ao fato de que durante o atraso $T_{0}$ a resposta impulsiva é nula, isto faz com que os coeficientes dos atrasadores correspondentes sejam nulos. Portanto não há necessidade de se utilizar a multiplicação $g_{j} x_{i}$ para estes primeiros atrasadores, nem mesmo as operações matemáticas do controle adaptativo. Somente para os sinais de saída dos atrasadores correspondentes à região de dispersão é que tais operações são necessárias, uma vez que só então $g_{i} \neq 0$. 
Explorando esse fato, a AMI usa um dispositivo, chamado "local loop delay estimator", que consegue estimar o atraso na ligação. Esta informação é enviada a um processador de controle que produz um atraso fixo igual ao valor estimado. Sua unidade de memória pode atrasar até $25,6 \mathrm{~ms}$. Entretanto, este atraso pode ser expandido em incrementos de $25 \mathrm{~ms}$ por adições de memórias de armazenamento [5]. Para a região de dispersão são utilizados atrasadores de $125 \mu \mathrm{s}$ cujas saídas fornecem sinais para as operações matemáticas de filtragem transversal e controle adaptativo. Por segurança, foi considerado um tempo de dispersão igual a $6 \mathrm{~ms}$. Isto acarreta a presença de $6 / 0,125=48$ atrasadores internos ao circuito integrado e, conseqüentemente, o mesmo número de operadores matemáticos.

Além disto, em circuitos digitais, prefere-se usar a aritmética seqüêncial. Com isto não é necessário usar 48 multiplicadores, integradores, e assim por diante, em paralelo, mas apenas um de cada tipo, trabalhando 48 vezes mais rápido que no caso paralelo. Esta rapidez é necessária para que todas as operações matemáticas sejam realizadas dentro de um período $T_{S}$. Isto requer um periodo de relógio muito menor que $T_{\mathbf{s}^{*}}$ Entretanto, economiza-se em quantidade de circuitos.

\section{Referências}

[1] M. M. Sondhi, "An Adaptive Echo Canceler", Bell System Technical Journal, vol. 46, ne 3, Março 1967, pp. 497-511.

[2] S.J. Campanella, H.G. Suyderhoud e M. Onufry, "Analysis of an Adaptive Impulse Response Echo Canceler", COMSAT Technical Review, vol. 2, nọ 2. Spring 1972, pp. 1-38.

[3] D.L. Duttweiler, "A Twelve-Channel Digital EchoCanceler", IEEE Transactions on Communications, vol. COM-26, no 5, Maio 1978, pp. 647-653.

[4] D. L. Duttweiler e Y.S. Chen, "A Single Chip VLSI Echo Canceler", Bell System Technical Journal, vol. 59, no 2, Fevereiro 1980, p.p. 149-160.

[5] "Echo Canceler Processor", Publicação Técnica da American Microsystems, Inc., Newport Beach, California, Estados Unidos, Setembro 1981. 
EDSON BENEDICTO R. FÉRIS concluiu o curso de Engenharia Eletrônica no Instituto Tecnológico da Aeronáutica (ITA) em 1959 e obteve os graus de mestre e doutor em Engenharia Elétrica na Universidade de São Paulo (USP), em 1971 e 1977, respectivamente. De 1960 até 1973 foi chefe do Laboratório de Telecomunicações da AEG Telefunken do Brasil e de 1973 até 1977 esteve na Fundação para Desenvolvimento Tecnológico de Engenharia (FDTE), onde foi coordenador técnico do Projeto SISCOM. Desde 1977 trabalha na Gerência de Transmissão do Centro de Pesquisa e Desenvolvimento (CPqD) da TELEBRÁS. 